\title{
Molecular Characterization of High-Risk Human Papillomavirus in Women in Bobo-Dioulasso, Burkina Faso
}

\author{
Ina Marie Angèle Traore, ${ }^{1,2}$ Théodora Mahoukèdè Zohoncon, ${ }^{1,2}$ Adama Dembele, ${ }^{3}$ \\ Florencia W. Djigma, ${ }^{1,2}$ Dorcas Obiri-Yeboah, ${ }^{4}$ Germain Traore, ${ }^{5}$ Moussa Bambara, ${ }^{3}$ \\ Charlemagne Ouedraogo, ${ }^{1,6,7}$ Yves Traore, ${ }^{8}$ and Jacques Simpore ${ }^{1,2,9}$ \\ ${ }^{1}$ Laboratory of Molecular Biology and Genetics (LABIOGENE), University of Ouagadougou, P.O. Box 7021, \\ Ouagadougou, Burkina Faso \\ ${ }^{2}$ Pietro Annigoni Biomolecular Research Center (CERBA), P.O. Box 364, Ouagadougou, Burkina Faso \\ ${ }^{3}$ Department of Gynecology, Obstetrics and Reproductive Medicine, Sourou Sanou Teaching Hospital, \\ P.O. Box 676, Bobo-Dioulasso, Burkina Faso \\ ${ }^{4}$ School of Medical Sciences, Microbiology Department, University of Cape Coast, Cape Coast, Ghana \\ ${ }^{5}$ Polyvalent Medical Center-Health and Reproduction, P.O. Box 1418, Bobo-Dioulasso, Burkina Faso \\ ${ }^{6}$ Yalgado Ouédraogo University Hospital (CHU/YO), P.O. Box 7022, Ouagadougou, Burkina Faso \\ ${ }^{7}$ UFR/SDS, University of Ouagadougou, P.O. Box 7021, Ouagadougou, Burkina Faso \\ ${ }^{8}$ Training and Research Unit in Life and Earth Science (UFR/SVT), University of Ouagadougou, P.O. Box 7021, \\ Ouagadougou, Burkina Faso \\ ${ }^{9}$ Faculty of Medicine, University Saint Thomas d'Aquin, P.O. Box 10212, Ouagadougou, Burkina Faso
}

Correspondence should be addressed to Jacques Simpore; jacques.simpore@yahoo.fr

Received 16 January 2016; Revised 14 June 2016; Accepted 23 June 2016

Academic Editor: Marcelo A. Soares

Copyright (C) 2016 Ina Marie Angèle Traore et al. This is an open access article distributed under the Creative Commons Attribution License, which permits unrestricted use, distribution, and reproduction in any medium, provided the original work is properly cited.

\begin{abstract}
High-risk human papillomavirus (HPV) is found in over $99 \%$ of cervical cancers. The aim of this study was to determine the prevalence of HPV in a population of women in Bobo-Dioulasso and to identify the high-risk types present in these women. From May to June, 2015, 181 women who came for consultation at the Souro Sanou University Hospital of Bobo-Dioulasso have been included in this study. Uterine endocervical swabs have been taken in these women. DNA obtained by extraction from the samples thus collected was used to determine the prevalence of high-risk human papillomavirus genotypes through real-time PCR. The age of the women ranged from 20 to 56 years with a mean of $35.3 \pm 8.1$ years. The prevalence of infection by high-risk HPV types was 25.4\% (46/181). The most common high-risk HPV genotypes were HPV 39 (18.5\%), HPV 52 (16.7\%), HPV 18 (14.8\%), and HPV 35 (13.0\%). HPV 16 which is included in the HPV vaccines was not found in the population studied. This type of study which is the first one in Bobo-Dioulasso has showed a high prevalence of genotypes HPV 39, HPV 52, and HPV 35 which are not yet covered by a vaccine.
\end{abstract}

\section{Introduction}

The human papillomavirus (HPV) infection is one of the most common sexually transmitted infections in the world $[1,2]$. In most cases, the infection may be unnoticed after a clearance period between 8 and 12 months [3]. The persistence of HPV infection with high oncogenetic risk is a condition for the occurrence of cervical precancerous and cancerous lesions [4]. When these lesions are not treated, some are likely to progress into cancer and HPV is found in $99.7 \%$ of cervical cancers [5]. With an incidence estimated at about 530,000 new cases and 275,000 deaths worldwide each year [6], cervical cancer is the leading cancer in women in sub-Saharan Africa and it remains a serious public health issue. While HPV 16 and HPV 18 genotypes are involved in approximately $70 \%$ of cancers of the cervix in the world [7], 
the distribution of the other genotypes follows geographic variation. This difference in the distribution of genotypes was found in women who were living in different regions of the same country [8]. In Burkina Faso, the study conducted by Zohoncon et al. in 2013 based on two previous studies in Ouagadougou $[9,10]$ showed that the high-risk HPV genotypes which were the most common were HPV 35 and HPV 52 [11]. In order to determine if the distribution of highrisk HPV genotypes was following the same trend in another city in Burkina Faso, we undertook this study. The aim of this study was to determine the prevalence of high-risk human papillomavirus in a population of women in Bobo-Dioulasso and to identify genotypes found in this city.

\section{Materials and Methods}

The study was conducted at the Souro Sanou University Hospital of Bobo-Dioulasso, the second city of Burkina Faso. From May to June, 2015, 181 women who came for consultation in the Department of Gynecology, Obstetrics, and Reproductive Medicine (DGOMR) have been included in the study. Pregnant women or women who have undergone hysterectomy or menstruating women have been excluded. Each woman answered a questionnaire to provide information on their socioeconomic status and behavioral and sexual habits. Samples have been taken through endocervical swabbing of the uterus using a sterile cotton swab. The samples thus obtained have been immersed in a transport medium which was provided with DNA-Sorb-A kit (Sacace Biotechnologies, Como, Italy) and kept at $-20^{\circ} \mathrm{C}$ until the DNA extraction. Immediately after sampling, screening for precancerous lesions was done for the women by visual inspection with acetic acid and Lugol's iodine (VIA/VILI).

This study has been approved by the Ethics Committee for Research in Health of Burkina Faso (Deliberation number 2014-9-110) and all the women have signed a consent form prior to participation.

The DNA extraction was made using DNA-Sorb-A kit (Sacace Biotechnologies, Como, Italy). The genotyping of high-risk HPV was made by real-time PCR using the kit "HPV Genotypes 14 Real-TM Quant," code V67-100FRT (Sacace Biotechnologies, Como, Italy), and the Sacycler-96 Real-Time PCR (Sacace Biotechnologies, Como, Italy). This genotyping is based on multiplex real-time amplification for each sample and the $\beta$-globin gene was used as internal control. The "HPV Genotypes 14 Real-TM Quant" made it possible to detect the following 14 high-risk HPV genotypes: $16,18,31,33,35,39,45,51,52,56,58,59,66$, and 68 .

The PCR program used was as follows: 1 cycle of $95^{\circ} \mathrm{C}$ for 15 minutes; 5 cycles of $95^{\circ} \mathrm{C}$ for 5 seconds, $60^{\circ} \mathrm{C}$ for 20 seconds, $72^{\circ} \mathrm{C}$ for 15 seconds; and 40 cycles of $95^{\circ} \mathrm{C}$ for 5 seconds, $60^{\circ} \mathrm{C}$ for 30 seconds, and $72^{\circ} \mathrm{C}$ for 15 seconds.

Data were entered and analyzed using the SPSS software in its 20.0 version and Epi Info 7. The Chi-square test was used for comparisons with a significant difference for $p<0.05$. Women under 30 years were considered as young women and these criteria were used in the study to group the women in age group: $\geq 30$ years old versus $<30$ years old.

\section{Results}

During the enrollment period in the Department of Gynecology, Obstetrics, and Reproductive Medicine (DGOMR), 181 women gave their consent to participate to this study. The main motivations for consultation were leucorrhea and pruritus (41, 4\%), cervical cancer screening (18, 2\%), abdominalpelvic pain $(11,6 \%)$, dysmenorrhea $(7,2 \%)$, contraception needs $(6,1 \%)$, maternity desire $(4,4 \%)$, breast pain $(3,9 \%)$, and others $(7,2 \%)$.

3.1. The Sociodemographic Characteristics of the Women in the Study. The sociodemographic, sexual, and behavioral characteristics of the study population are given in Table 1. The women's age ranged from 20 to 56 years with a mean of $35.3 \pm 8.1$ years; and $50.8 \%$ of the women were under 35 . The majority of women $(84 \%)$ were married or lived with a partner. Housewives and women working in the informal sector accounted for $68.5 \%$ and $15.5 \%$, respectively; only $8.8 \%$ of the women were employed and $7.2 \%$ were either high school students or university students. As for education level, $34.3 \%$ and $1.1 \%$ of the women had reached high school and university, respectively. The others were either illiterate (35.9\%) or had primary level $(28.7 \%)$. The age of the women at first sexual intercourse ranged from 13 to 27 years with a mean of $18.57 \pm 2.2$ years. Among all women, only $6.6 \%$ had never been pregnant. The other had at least one pregnancy in their lives and $18.8 \%$ of them had more than 5 children. More than half of the women (67.4\%) had never had an abortion while the others had at least one miscarriage. In addition, $66.3 \%$ of the women were not using contraception at the time of inclusion in the study.

3.2. The Prevalence of High-Risk HPV Genotypes. In this study, all tested samples were positive for $\beta$-globin gene. The results of HPV research show that $25.4 \%$ (46/181) of the women were infected with high-risk HPV. The number of HPV genotypes per woman ranged from 1 to 3 , and in considering multiple infections, we counted in total 54 genotypes among the 46 women infected with HPV. Figure 1 shows the distribution of these genotypes among which the most common ones were HPV 39 (18.5\%), HPV 52 (16.7\%), HPV 18 (14.8\%), and HPV 35 (13.0\%). HPV 16 and HPV 33 were not found in the women included in this study.

3.3. Distribution of Genotypes Based on the Type of Infection. $84.8 \%$ of the women infected with HPV were carriers of a single genotype. There was $15.2 \%$ (7/46) of multiple infections including 6 multiple infections with 2 genotypes and 1 multiple infection with 3 genotypes (Table 2). This table also shows that the distribution of genotypes found in this study was not statistically different among the women whether they are over or under 30 years of age $(p=0.335)$.

3.4. Potential Risk Factors Associated with the Carrying of $H P V$. In Table 3, we analyzed association between the HPV infection and age, age at first sexual intercourse, parity, the use of oral contraception, and the VIA/VILI result. On the 181 women of the study, visual screening for precancerous lesions was not done for 2 of them. The results showed that 9/179 
TABLE 1: Sociodemographic, sexual, and behavioral characteristics of women included in the study.

\begin{tabular}{|c|c|c|}
\hline Characteristics & Number & $\%$ \\
\hline \multicolumn{3}{|l|}{ Age groups in years } \\
\hline $20-24$ & 14 & 7.7 \\
\hline $25-34$ & 78 & 43.1 \\
\hline$\geq 35$ & 89 & 49.2 \\
\hline \multicolumn{3}{|l|}{ Level of education } \\
\hline Illiterate & 65 & 35.9 \\
\hline Primary & 52 & 28.7 \\
\hline Secondary & 62 & 34.3 \\
\hline University & 02 & 1.1 \\
\hline \multicolumn{3}{|l|}{ Marital status } \\
\hline Married or lives with a partner & 152 & 84.0 \\
\hline Single & 16 & 08.8 \\
\hline Widow & 11 & 6.1 \\
\hline Divorced & 02 & 1.1 \\
\hline \multicolumn{3}{|l|}{ Number of sexual partners } \\
\hline 0 & 13 & 7.2 \\
\hline 1 & 164 & 90.6 \\
\hline$\geq 2$ & 04 & 2.2 \\
\hline \multicolumn{3}{|l|}{ Occupation } \\
\hline Housewife & 124 & 68.5 \\
\hline Pupil or student & 13 & 7.2 \\
\hline Employee & 16 & 8.8 \\
\hline Informal sector & 28 & 15.5 \\
\hline \multicolumn{3}{|l|}{ Use of contraception } \\
\hline No/natural method & 120 & 66.3 \\
\hline Yes & 61 & 33.7 \\
\hline \multicolumn{3}{|l|}{ Age at the first sexual intercourse } \\
\hline $13-17$ & 48 & 30 \\
\hline $18-24$ & 109 & 68.1 \\
\hline$\geq 25$ & 03 & 1.9 \\
\hline Unanswered & 21 & \\
\hline \multicolumn{3}{|l|}{ Number of pregnancies } \\
\hline 0 & 12 & 6,6 \\
\hline $1-2$ & 64 & 35.4 \\
\hline $3-5$ & 71 & 39.2 \\
\hline $6-11$ & 34 & 18.8 \\
\hline \multicolumn{3}{|l|}{ Number of abortions } \\
\hline 0 & 122 & 67.4 \\
\hline 1 & 40 & 22.1 \\
\hline 2 & 13 & 7.2 \\
\hline$\geq 3$ & 06 & 3.3 \\
\hline
\end{tabular}

women or $5.0 \%$ were positive to VIA/VILI. This study found no statistical association between HPV infection and the risk factors analyzed.

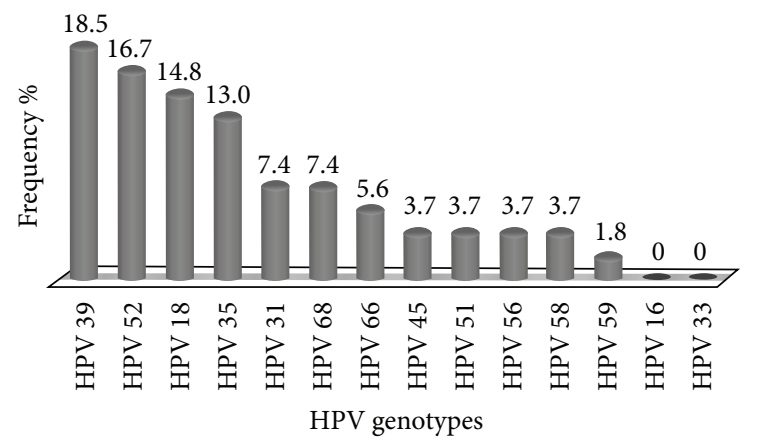

FIGURE 1: Frequency of high-risk HPV genotypes found among the women in the study.

\section{Discussion}

This study shows the characteristics of the high-risk HPV infection in a general population of women in BoboDioulasso. To the best of our knowledge, in Bobo-Dioulasso, it is the first study on a general population of women concerning the HPV. Indeed, the other studies conducted in this city have focused on a particular group of women who are sex workers $[12,13]$. However, the majority of women $(90.6 \%)$ in the current study had a single sexual partner and $7.2 \%$ of the women reported no sexual activity at the time of inclusion in the study. The women in this study had a mean age of 35.3 years; Ouedraogo et al. in 2011 [10] had also found an average age of over 30 years among women attending gynecological consultation in Ouagadougou. But unlike this study which counted $38.3 \%$ of housewives, more than half $(68.5 \%)$ of the women in our study were housewives.

The prevalence of $25.4 \%$ of high-risk HPV obtained in this study is high compared to the worldwide prevalence of HPV infection estimated to be around 11-12\% [14]. Several studies suggest a higher prevalence of oncogenic HPV type in sub-Saharan Africa compared to elsewhere [15-17] and the average prevalence reported in this part of Africa was $24 \%$ [14]. The prevalence of high-risk HPV in this study is in line with the $23.2 \%$ reported in Thiès, in Senegal [8], and the $23 \%$ in rural women in Mali [18]. In two studies conducted in Ouagadougou, Burkina Faso [11, 19], the high-risk HPV prevalence was $30.2 \%$ and $41.5 \%$, higher than which was found in the present study. The last study conducted in Ouagadougou focused on adolescent girls and this could explain the difference in the prevalence compared to this study. Indeed, the average age of our population was 35.3 years while it has been indicated that the prevalence of high-risk HPV infection was maximum before the age of 30 years $[20,21]$.

HPV 39 (18.5\%), HPV 52 (16.7\%), HPV 18 (14.8\%), and HPV 35 (13.0\%) were the most common genotypes in this study. The frequency of the other genotypes found ranged from 7.4 to $1.8 \%$. HPV 39 was not commonly found at the top of the most common genotypes in the literature. But it was part of the 3 most common genotypes in women with Atypical Squamous Cells of Undetermined Significance (ASCUS) [22]. A high prevalence of HPV 52 was also observed in Japan [23], Tanzania [24], and Burkina Faso [11, 
TABLE 2: HPV genotypes distribution according to the association with multiple or isolated infections and depending on the age.

\begin{tabular}{|c|c|c|c|}
\hline HPV genotypes & $\begin{array}{c}\text { Age }<30 \\
n(\%)\end{array}$ & $\begin{array}{c}\text { Age } \geq 30 \\
n(\%)\end{array}$ & $\begin{array}{l}\text { Total } \\
n(\%)\end{array}$ \\
\hline \multicolumn{4}{|c|}{ Genotypes associated with isolated infections } \\
\hline HPV 18 & - & $6(13.0)$ & $6(13.0)$ \\
\hline HPV 31 & - & $3(6.5)$ & $3(6.5)$ \\
\hline HPV 35 & $3(6.5)$ & $3(6.5)$ & $6(13.0)$ \\
\hline HPV 39 & $3(6.5)$ & $6(13.0)$ & $9(16.7)$ \\
\hline HPV 45 & - & $1(2.2)$ & $1(2.2)$ \\
\hline HPV 51 & - & $1(2.2)$ & $1(2.2)$ \\
\hline HPV 52 & $2(4.4)$ & $3(6.5)$ & $5(10.9)$ \\
\hline HPV 56 & $1(2.2)$ & - & $1(2.2)$ \\
\hline HPV 58 & - & $2(4.3)$ & $2(4.4)$ \\
\hline HPV 66 & - & $3(6.5)$ & $3(6.5)$ \\
\hline HPV 68 & $1(2.2)$ & $1(2.2)$ & $2(4.4)$ \\
\hline Total 1 & $10(21.8)$ & $29(63.0)$ & $39(84.8)$ \\
\hline \multicolumn{4}{|c|}{ Genotypes associated with multiple infections } \\
\hline HPV $18+52$ & $1(2.2)$ & - & $1(2.2)$ \\
\hline HPV $18+68$ & - & $1(2.2)$ & $1(2.2)$ \\
\hline HPV $39+51$ & $1(2.2)$ & - & $1(2.2)$ \\
\hline HPV $45+52$ & - & $1(2.2)$ & $1(2.2)$ \\
\hline HPV 56+52 & - & $1(2.2)$ & $1(2.2)$ \\
\hline HPV 59+68 & $1(2.2)$ & - & $1(2.2)$ \\
\hline HPV $31+35+52$ & - & $1(2.2)$ & $1(2.2)$ \\
\hline Total 2 & $3(6.5)$ & $4(8.7)$ & $7(15.2)$ \\
\hline General total & $13(28.3)$ & $33(71.7)$ & $46(100)$ \\
\hline
\end{tabular}

TABLE 3: Factors associated with oncogenic HPV infection.

\begin{tabular}{|c|c|c|c|c|}
\hline & $\begin{array}{l}\text { HPV- } \\
n=135\end{array}$ & $\begin{array}{l}\text { HPV+ } \\
n=46\end{array}$ & $\begin{array}{c}\text { Total } \\
n=181\end{array}$ & $p^{*}$ \\
\hline Age group, $n(\%)$ & & & & 0.99 \\
\hline$<30$ & $40(29.6)$ & $13(28.3)$ & 53 & \\
\hline$\geq 30$ & $95(70.4)$ & $33(71.7)$ & 128 & \\
\hline Age at first sexual intercourse, $n(\%)$ & & & & 0.88 \\
\hline$<16$ & $12(8.9)$ & $05(10.9)$ & 17 & \\
\hline$\geq 16$ & $108(80)$ & $35(76.1)$ & 143 & \\
\hline Unanswered & $15(11.1)$ & $06(13.0)$ & 21 & \\
\hline Parity, $n(\%)$ & & & & 0.76 \\
\hline Nulliparous & $9(6.7)$ & $3(6.5)$ & 12 & \\
\hline Primiparous & $18(13.3)$ & $7(15.2)$ & 25 & \\
\hline Multiparous & $108(80)$ & $36(78.3)$ & 144 & \\
\hline Use of oral contraception, $n(\%)$ & & & & 0.09 \\
\hline Yes & $16(11.8)$ & $01(2.2)$ & 17 & \\
\hline No & $119(88.2)$ & $45(97.8)$ & 164 & \\
\hline VIA/VILI result, $n(\%)^{* *}$ & & & & 0.85 \\
\hline VIA/VILI- & $127(74.7)$ & $43(25.3)$ & 170 & \\
\hline VIA/VILI+ & $7(77.8)$ & $2(22.2)$ & 9 & \\
\hline
\end{tabular}

${ }^{*} p$ value for difference in characteristic according to HR-HPV status.

${ }^{* *}$ VIA/VILI result was available for $n=179$ women. 
19]. In Didelot-Rousseau et al's [12] study conducted in BoboDioulasso, HPV 52 was the most frequent type followed by HPV 35. These authors examined both high and low risk HPV types; HPV 16 with HPV 18 were, respectively, the 6th and the 8 th most frequent genotype found in women. The prevalence of HPV 18 obtained in the present study was $14.8 \%$; it is greater than that reported in Bobo-Dioulasso (6.4\%) [12] and even in Colombia (7.1\%) [25]. However, it is less than the value found in South African women (21.0\%) [24]; the authors of that study showed a regional difference in the distribution of HPV 18.

HPV 16 and HPV 18 are the high-risk genotypes included in the vaccines available to fight against cervical cancer. HPV 16 was not found in this study. Some studies had already shown a low prevalence of HPV 16 in some African countries compared to other high-risk HPV genotypes [1, 11, 19, 26]. However, HPV 16 was found as the most common genotype in the world especially in Europe [27], the USA [28], and North Africa [29].

In this study, the carrying of the high-risk HPV was not associated with risk factors such as the age at first sexual intercourse before the age of 16 [30], the use of oral contraception, the parity, or the VIA/VILI result. Human Immunodeficiency Virus (HIV) infection may also be considered as a risk factor that influences HPV infection. Indeed, HIV upregulates HPV replication [31] and HPV persistence [32] leading to the evolution of HPV infection from high-risk lesions to uterine cervix cancer. It is known that HIV-positive women have higher proportion of HPV infection than HIVnegative and HIV infection could have an impact on HPV genotype distribution [33].

In the present study, we did not investigate women's HIV status but it is known that HIV prevalence for the general population of Bobo-Dioulasso is 1.4\% [34]. This value is within the highest prevalence of Burkina Faso where adults HIV prevalence is estimated to be less than $1 \%$ [35]. The relative high HIV prevalence for the general population of Bobo-Dioulasso could be a risk factor for HPV infection in this population. Multiple infections with several HPV types are common among HIV infected women. In this study, women had little multiple infections (15.2\%) with high-risk HPV compared to $90,1 \%$ reported in HIV infected women by Zohoncon et al. [11]. The present study percentage is closer to the $12.3 \%$ of multiple infections reported in women attending gynecological consultation in Ouagadougou [10].

The results of this study could certainly not be generalized to the entire population of Bobo-Dioulasso but they helped to know the HPV genotypes present in a population of women in this city. This is of real significance in the sense that the data obtained in a city other than Ouagadougou will be used to have a broader overview of the distribution of high-risk $\mathrm{HPV}$ genotypes in Burkina Faso.

\section{Conclusion}

The most common genotypes in this study were HPV 39, HPV 52, HPV 18, and HPV 35. These results are consistent with those of other studies conducted in Burkina Faso, which showed that there was a predominance of high-risk HPV other than HPV 16 and HPV 18. The future vaccine trials should therefore consider these other high-risk genotypes in order to expand prevention through anti-HPV vaccination.

\section{Competing Interests}

The authors declare there are no competing interests regarding the publication of this paper.

\section{Acknowledgments}

The authors are thankful to CERBA/LABIOGENE at the University of Ouagadougou; the Souro Sanou hospital; the Center Muraz of Bobo-Dioulasso; and Professor Annie Robert of the Catholic University of Louvain in Belgium. They express their gratitude to the Italian Episcopal Conference and to WAEMU/PACER2.

\section{References}

[1] L. Bruni, M. Diaz, X. Castellsagué, E. Ferrer, F. X. Bosch, and S. de Sanjosé, "Cervical human papillomavirus prevalence in 5 continents: meta-analysis of 1 million women with normal cytological findings," The Journal of Infectious Diseases, vol. 202, no. 12, pp. 1789-1799, 2010.

[2] E. F. Dunne, E. R. Unger, M. Sternberg et al., "Prevalence of HPV infection among females in the United States," The Journal of the American Medical Association, vol. 297, no. 8, pp. 813-819, 2007.

[3] E. L. Franco, L. L. Villa, J. P. Sobrinho et al., "Epidemiology of acquisition and clearance of cervical human papillomavirus infection in women from a high-risk area for cervical cancer," The Journal of Infectious Diseases, vol. 180, no. 5, pp. 1415-1423, 1999.

[4] N. Muñoz, X. Castellsagué, A. B. de González, and L. Gissmann, "Chapter 1: HPV in the etiology of human cancer," Vaccine, vol. 24, supplement 3, pp. S1-S10, 2006.

[5] J. M. Walboomers, M. V. Jacobs, M. M. Manos et al., "Human papillomavirus is a necessary cause of invasive cervical cancer worldwide," The Journal of Pathology, vol. 189, no. 1, pp. 12-19, 1999.

[6] M. Arbyn, X. Castellsagué, S. de sanjosé et al., "Worldwide burden of cervical cancer in 2008," Annals of Oncology, vol. 22, no. 12, pp. 2675-2686, 2011.

[7] N. Li, S. Franceschi, R. Howell-Jones, P. J. F. Snijders, and G. M. Clifford, "Human papillomavirus type distribution in 30,848 invasive cervical cancers worldwide: variation by geographical region, histological type and year of publication," International Journal of Cancer, vol. 128, no. 4, pp. 927-935, 2011.

[8] E. H. S. Mbaye, T. Gheit, A. Dem et al., "Human papillomavirus infection in women in four regions of Senegal," Journal of Medical Virology, vol. 86, no. 2, pp. 248-256, 2014.

[9] F. W. Djigma, C. Ouédraogo, D. S. Karou et al., "Prevalence and genotype characterization of human papillomaviruses among HIV-seropositive in Ouagadougou, Burkina Faso," Acta Tropica, vol. 117, no. 3, pp. 202-206, 2011.

[10] C. M. R. Ouedraogo, F. W. Djigma, C. Bisseye et al., "Epidemiology, characterization of genotypes of human papillomavirus in a population of women in Ouagadougou," Journal de Gynecologie, Obstetrique et Biologie de la Reproduction, vol. 40, no. 7, pp. 633638, 2011. 
[11] T. M. Zohoncon, C. Bisseye, F. W. Djigma et al., "Prevalence of HPV high-risk genotypes in three cohorts of women in Ouagadougou (Burkina Faso)," Mediterranean Journal of Hematology and Infectious Diseases, vol. 5, no. 1, Article ID e2013059, 6 pages, 2013.

[12] M.-N. Didelot-Rousseau, N. Nagot, V. Costes-Martineau et al., "Human papillomavirus genotype distribution and cervical squamous intraepithelial lesions among high-risk women with and without HIV-1 infection in Burkina Faso," British Journal of Cancer, vol. 95, no. 3, pp. 355-362, 2006.

[13] A. J. Low, T. Clayton, I. Konate et al., "Genital warts and infection with human immunodeficiency virus in high-risk women in Burkina Faso: A Longitudinal Study," BMC Infectious Diseases, vol. 11, article 20, 2011.

[14] D. Formana, C. de Martel, C. J. Lacey et al., "Global burden of human papillomavirus and related diseases," Vaccine, vol. 30, supplement 5, pp. F12-F23, 2012.

[15] X. Castellsagué, C. Menéndez, M.-P. Loscertales et al., "Human papillomavirus genotypes in rural Mozambique," The Lancet, vol. 358, no. 9291, pp. 1429-1430, 2001.

[16] G. M. Clifford, S. Gallus, R. Herrero et al., "Worldwide distribution of human papillomavirus types in cytologically normal women in the International Agency for Research on Cancer HPV prevalence surveys: a pooled analysis," The Lancet, vol. 366, no. 9490, pp. 991-998, 2005.

[17] P. Mayaud, H. A. Weiss, C. J. N. Lacey, D. K. Gill, and D. C. W. Mabey, "Genital human papillomavirus genotypes in northwestern Tanzania," Journal of Clinical Microbiology, vol. 41, no. 9, pp. 4451-4453, 2003.

[18] N. H. Schluterman, S. O. Sow, C. B. Traore et al., "Differences in patterns of high-risk human papillomavirus infection between urban and rural low-resource settings: cross-sectional findings from Mali," BMC Women's Health, vol. 13, no. 1, article 4, pp. 1-9, 2013.

[19] C. M. R. Ouédraogo, R. M. L. Rahimy, T. M. Zohoncon et al., "Epidemiology and characterization of high-risk genotypes of human Papillomavirus in a population of sexually active adolescents in Ouagadougou," Journal de Gynecologie Obstetrique et Biologie de la Reproduction, vol. 44, no. 8, pp. 715-722, 2015.

[20] E. S. Cibas, X. Hong, C. P. Crum, and S. Feldman, "Age-specific detection of high risk HPV DNA in cytologically normal, computer-imaged ThinPrep Pap samples," Gynecologic Oncology, vol. 104, no. 3, pp. 702-706, 2007.

[21] M. V. Jacobs, J. M. M. Walboomers, P. J. F. Snijders et al., "Distribution of 37 mucosotropic HPV types in women with cytologically normal cervical smears: the age-related patterns for high-risk and low-risk types," International Journal of Cancer, vol. 87, no. 2, pp. 221-227, 2000.

[22] Y.-C. Chiang, W.-F. Cheng, Y.-L. Chen et al., "High-risk human papillomavirus, other than type $16 / 18$, in predominantly older Taiwanese women with high-grade cervical preinvasive lesions," Taiwanese Journal of Obstetrics \& Gynecology, vol. 52, no. 2, pp. 222-226, 2013.

[23] K. Takehara, T. Toda, T. Nishimura et al., "Human papillomavirus types 52 and 58 are prevalent in uterine cervical squamous lesions from Japanese women," Pathology Research International, vol. 2011, Article ID 246936, 7 pages, 2011.

[24] J. A. Dols, G. Reid, J. M. Brown et al., "HPV type distribution and cervical cytology among HIV-positive Tanzanian and South African women," ISRN Obstetrics and Gynecology, vol. 2012, Article ID 514146, 5 pages, 2012.
[25] A. M. Bedoya, A. M. Gaviria, A. Baena et al., "Age-specific seroprevalence of human papillomavirus 16,18,31, and 58 in women of a rural town of Colombia," International Journal of Gynecological Cancer, vol. 22, no. 2, pp. 303-310, 2012.

[26] T. Sagna, F. Djigma, M. Zeba et al., "Human papillomaviruses prevalence and genital co-infections in HIV-seropositive women in Ouagadougou (Burkina Faso)," Pakistan Journal of Biological Sciences, vol. 13, no. 19, pp. 951-955, 2010.

[27] W. A. Tjalma, X. B. Trinh, M. Rosenlund et al., "A crosssectional, multicentre, epidemiological study on human papillomavirus (HPV) type distribution in adult women diagnosed with invasive cervical cancer in Belgium," Facts, Views \& Vision in ObGyn, vol. 7, no. 2, pp. 101-108, 2015.

[28] J. Monsonego, J. T. Cox, C. Behrens et al., "Prevalence of highrisk human papilloma virus genotypes and associated risk of cervical precancerous lesions in a large U.S. screening population: data from the ATHENA trial," Gynecologic Oncology, vol. 137, no. 1, pp. 47-54, 2015.

[29] H. Guettiti, E. Ennaifer, L. Attia et al., "Pre-vaccination prevalence and genotype distribution of human papillomavirus infection among women from urban Tunis: a cross-sectional study," Asian Pacific Journal of Cancer Prevention, vol. 15, no. 21, pp. 9361-9365, 2014.

[30] A. A. Ribeiro, M. C. Costa, R. R. F. Alves et al., "HPV infection and cervical neoplasia: associated risk factors," Infectious Agents and Cancer, vol. 10, no. 1, article 16, 2015.

[31] M. L. Tornesello, F. M. Buonaguro, E. Beth-Giraldo, and G. Giraldo, "Human immunodeficiency virus type 1 tat gene enhances human papillomavirus early gene expression," Intervirology, vol. 36, no. 2, pp. 57-64, 1993.

[32] A.-B. Moscicki, J. H. Ellenberg, S. Farhat, and J. Xu, "Persistence of human papillomavirus infection in HIV-infected and uninfected adolescent girls: risk factors and differences, by phylogenetic type," Journal of Infectious Diseases, vol. 190, no. 1, pp. 37-45, 2004.

[33] M. F. D. Baay, E. F. Kjetland, P. D. Ndhlovu et al., "Human papillomavirus in a rural community in Zimbabwe: the impact of HIV co-infection on HPV genotype distribution," Journal of Medical Virology, vol. 73, no. 3, pp. 481-485, 2004.

[34] INSD, Prévalence du VIH au Burkina Faso: résultats de l'EDSBF-MICS IV 2010, http://www.insd.bf/n/contenu/autres_ publications/edsbf-micsIV_depliant_VIH.pdf.

[35] ONUSIDA, Estimations VIH et SIDA 2014, http://www.unaids .org/fr/regionscountries/countries/burkinafaso. 


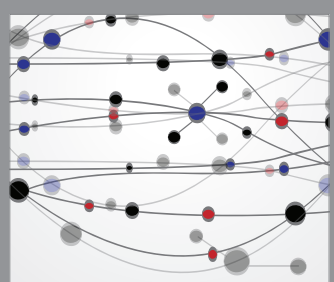

The Scientific World Journal
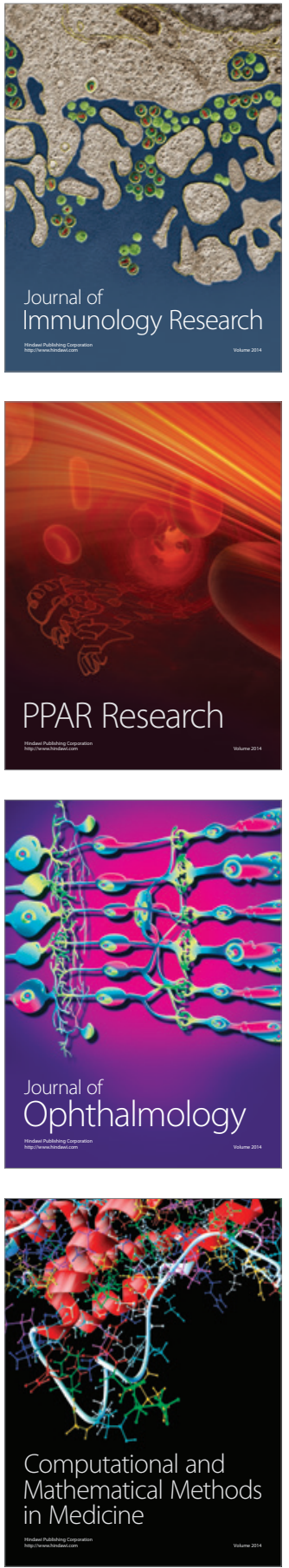

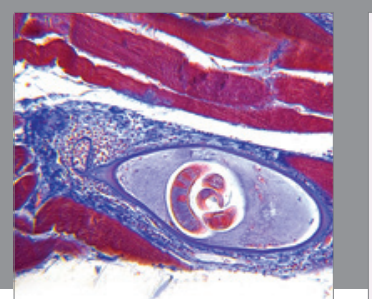

Gastroenterology Research and Practice

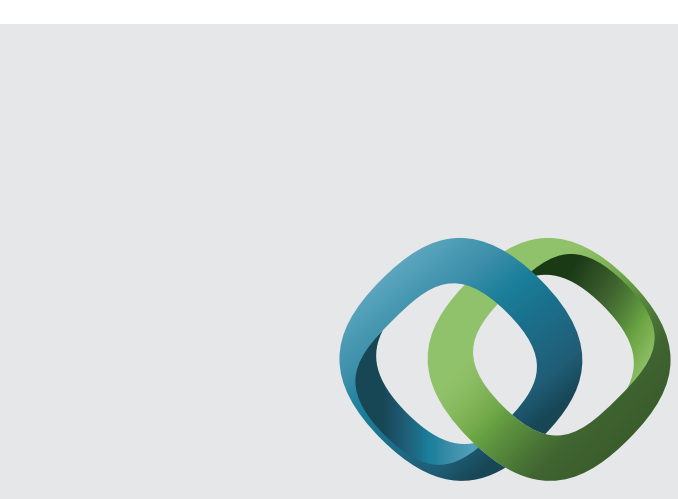

\section{Hindawi}

Submit your manuscripts at

http://www.hindawi.com
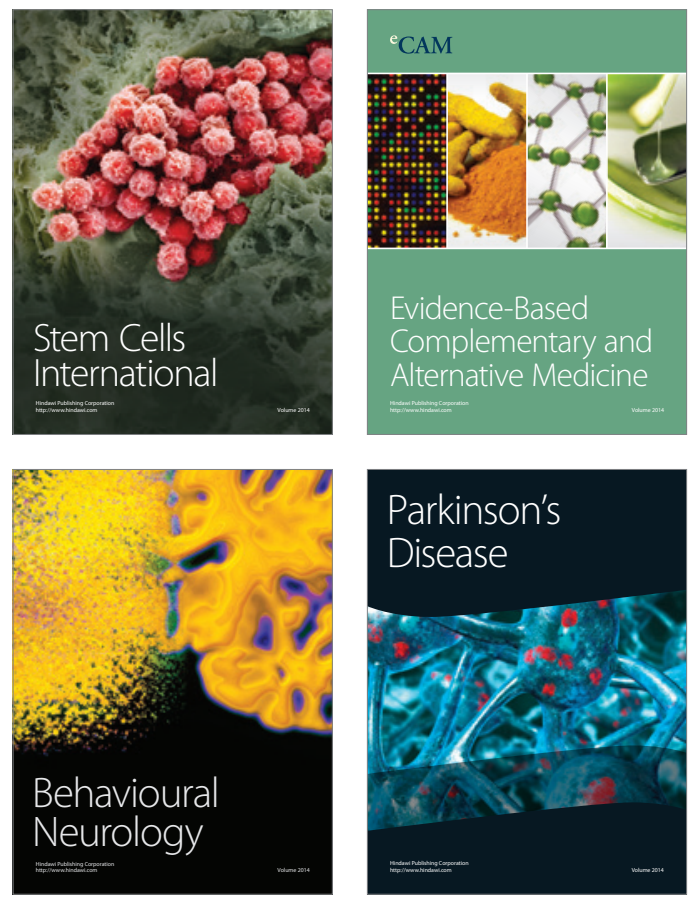
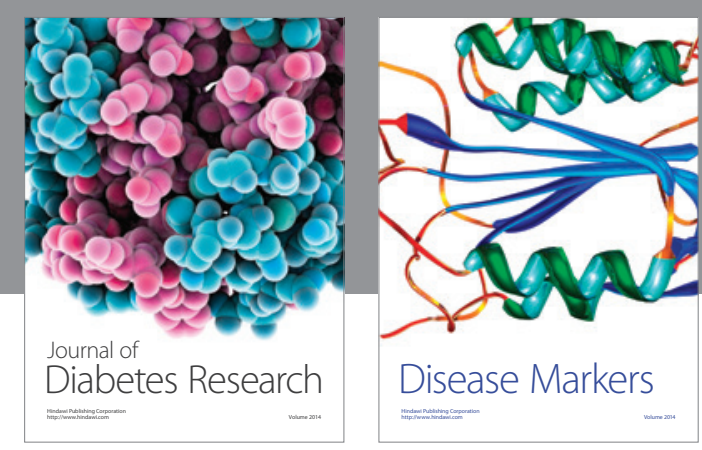

Disease Markers
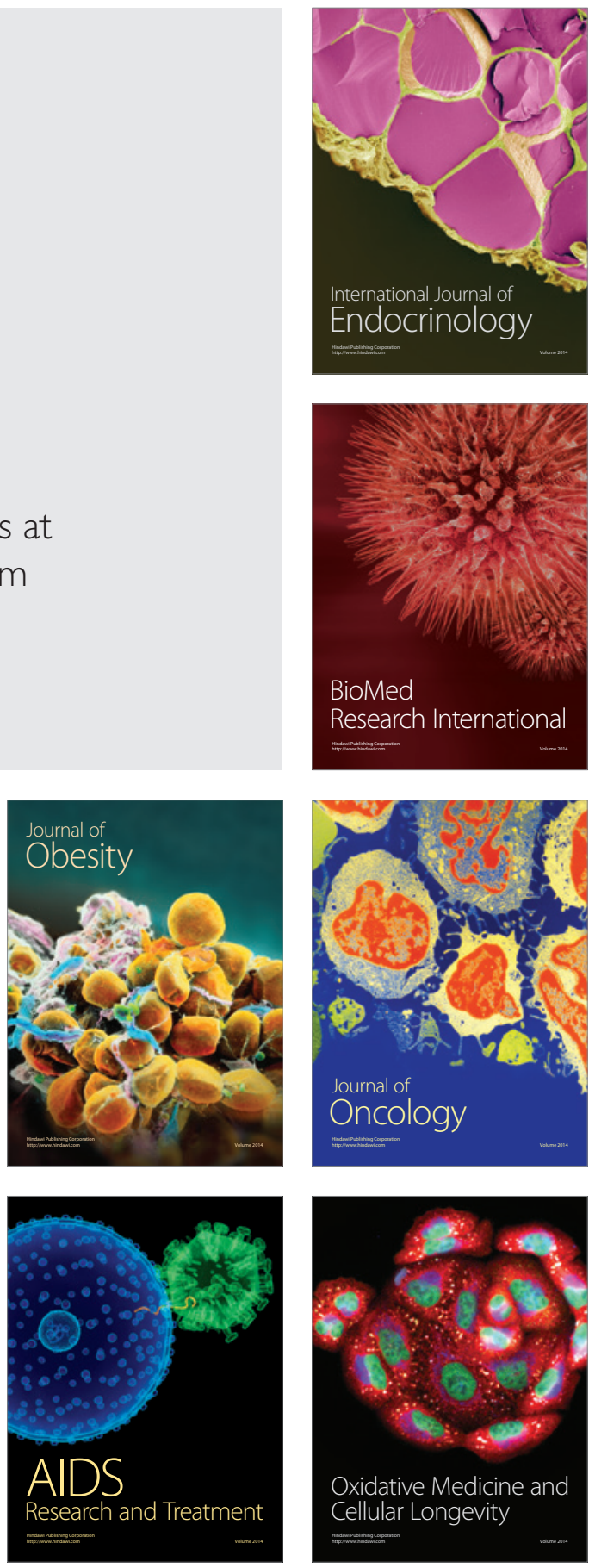